\title{
Neurofuzzy Model Based Predictive Control for thermal batch processes
}

\author{
J. M. Escaño*,a, C. Bordons ${ }^{\mathrm{a}}$, C. Vilas ${ }^{\mathrm{b}}$, M. R. García ${ }^{\mathrm{b}}$, A. A. Alonso ${ }^{\mathrm{b}}$ \\ ${ }^{a}$ Department of Systems Engineering and Automatic Control. University of Seville \\ (USE), Spain \\ ${ }^{b}$ Process Engineering Group. Institute for Marine Research. Spanish Council for \\ Scientific Research (CSIC), Vigo, Spain
}

\begin{abstract}
In many cases, it is difficult to derive a precise mathematical model, based on first principles, for a given process. Besides, the computation of the solution of models obtained through this methology may require a large computational effort making them useless for real time tasks like control or optimization. Neurofuzzy modelling, which permits an easy way to derive successful models, is a good alternative which can be employed to overcome such limitations. In this paper, together with the neurofuzzy modelling, several strategies based on non-linear predictive control are presented. The low computational cost associated with neurofuzzy models and controllers makes them suitable candidates to be implemented into industrial Programmable Logic Controllers (PLC). Both the model and controllers are validated and implemented in a pilot plant for the thermal sterilization of solid canned food in steam retorts and based on the results, a comparison between the different predictive control strategies is presented.
\end{abstract}

Key words: Neurofuzzy, Non-linear MPC, thermal processes

\section{Introduction}

Nowadays, predictive control is considered a well established technology in many fields, especially in industrial processes. Its efficiency has been demon-

\footnotetext{
*Corresponding author.

Email address: jmesca@cartuja.us.es (J. M. Escaño)
} 
strated over the last few years. In general, most applications of predictive control are based on linear models, which present good results especially if they work around a duty point [1]. However, there are many applications where the region of operation and/or the degree of "non-linearity" of the system reduce the prediction capabilities of linear models thus leading to a poor control quality. In such cases, Non Linear Model Predictive Control (NMPC) is a suitable option.

Although the number of applications of NMPC is limited, its potential is enormous. The possibility of dealing with nonlinear dynamics is the main advantage over MPC. However, developing precise nonlinear models from first principles may be a difficult task in many complex processes. Another disadvantage is that the optimizer solution in non-linear Predictive Control is a non-convex problem and a large computational effort may be required to obtain the solution. This is especially relevant when dealing with real time tasks due to the low computational capabilities of most platforms for industrial control based on PLCs (Programmable Logic Controllers).

Fuzzy models permit explicit solutions of the optimization (without restrictions) with a low computational cost. It should be remarked that an NMPC procedure based on Fuzzy models could be implemented on small platforms like PLCs. In this paper, neurofuzzy modelling has been used having a Fuzzy Inference System (FIS) as a model for NMPC. Fuzzy control has been applied successfully in many industrial processes [2]. It has required a special part of the IEC 1131[4] standard, which is about industrial PLC. Many groups have been involved in this part, leading to IEC 1131-7 [3]. In this part, a set of functions to program fuzzy control applications, is defined. This set of functions is named FCL (Fuzzy Control Language). Function blocks defined on FCL may be used in other languages established by IEC 1131-3. FCL functions are defined with the following names: Fuzzification, Defuzzification and Ruleblock [3]. The norm establishes, in addition, a set of optional parameters for them. Within the scope of the norm, programmers have a easy way to implement NMPC.

The sterilization of solid food in steam retorts has been chosen as a benchmark because this system presents a highly nonlinear behaviour. In addition, the operation needs to be guided by the achievement of strict requirements on microorganisms thermal destruction while maintaining the product under acceptable organoleptic specifications. Such goals must be attained despite a number of undesirable disturbances acting within the process like sudden steam temperature shut down situations due to boiler overload (excessive 
steam demand from different retorts). The process is also subject to a considerable degree of parameter uncertainties and to some extent also to a lack of accurate dynamic models (structural uncertainty): many simplifications like spatial homogeneity or isotropy assumptions are considered in order to obtain tractable models. All these issues make the plant be a good testbed for the illustration of the capabilities of the nonlinear predictive control strategies based on fuzzy models quoted before. Two different techniques have been developed using neurofuzzy models with Generalized Predictive Control $(G P C)[5]$ and applied to control the thermal sterilization process in steam retorts.

This paper is organized as follows. In section 2, a description of the sterilization of food plant is presented. In section 3, the neurofuzzy model of a Food Thermal Processing Application is described. In section 4, three MPC strategies for autoclave's temperature control are presented. The practical implementation of strategies is discussed in section 5. Finally, the conclusions derived from the results will be commented on section 6 .

\section{The sterilization of solid food plant}

A schematic representation of the pilot plant is depicted in Figure 1. This unit belongs to IIM-CSIC (Instituto de Investigaciones Marinas-Consejo Superior de Investigaciones Cientificas, Spain). The retort contains the product to be sterilized, usually consisting of a number of cans with the same specifications (geometry, size and type of food). Once in the retort, the load will be subjected to a time-temperature sterilization profile previously "designed" so to ensure a given lethality (a parameter related to the degree of reduction in a reference pathogen microorganism) while preserving in as much as possible the product quality. The specified profile is enforced by regulating the flow of a stream of saturated steam produced by the boiler. Such stream will first (in the venting stage, the first of the three stages of a sterilization cycle) be used to remove the air present in the retort, thus ensuring that the cans will be heated under the condition of a saturated steam. Such part of the operation, which is where sterilization is really carried out, is known as heating. During this stage, the product is usually kept at a given constant working temperature for a certain (predefined) time that will ensure the achievement of the desired microbiological lethality. At the critical point (the coldest point/point of least lethality inside the product), lethality is 
defined as follows:

$$
F_{0}=\int_{0}^{t} 10^{\frac{T_{r e f}^{k}-T\left(r_{0}\right)}{Z}} d t
$$

where z represents a kinetic parameter, $T_{r e f}^{k}$ its associated temperature and $T\left(r_{0}\right)$ the temperature at the critical point. It is pointed out that due to the exponential type relationship between temperature and lethality (equation 1), a fine temperature control is needed along this stage in order to avoid disturbances that could result in a serious over-processing of the product.

Finally, and in order to avoid over-processing and therefore quality losses, the product needs to be cooled as fast as possible. Cooling water is employed during this part of the operation as a means of fast cooling while compressed air will be introduced in the retort in order to compensate for sharp pressure drops. A complete description of the sterilization process and operation for different control schemes can be found in [6], [7], [8] and [9]. In this pilot

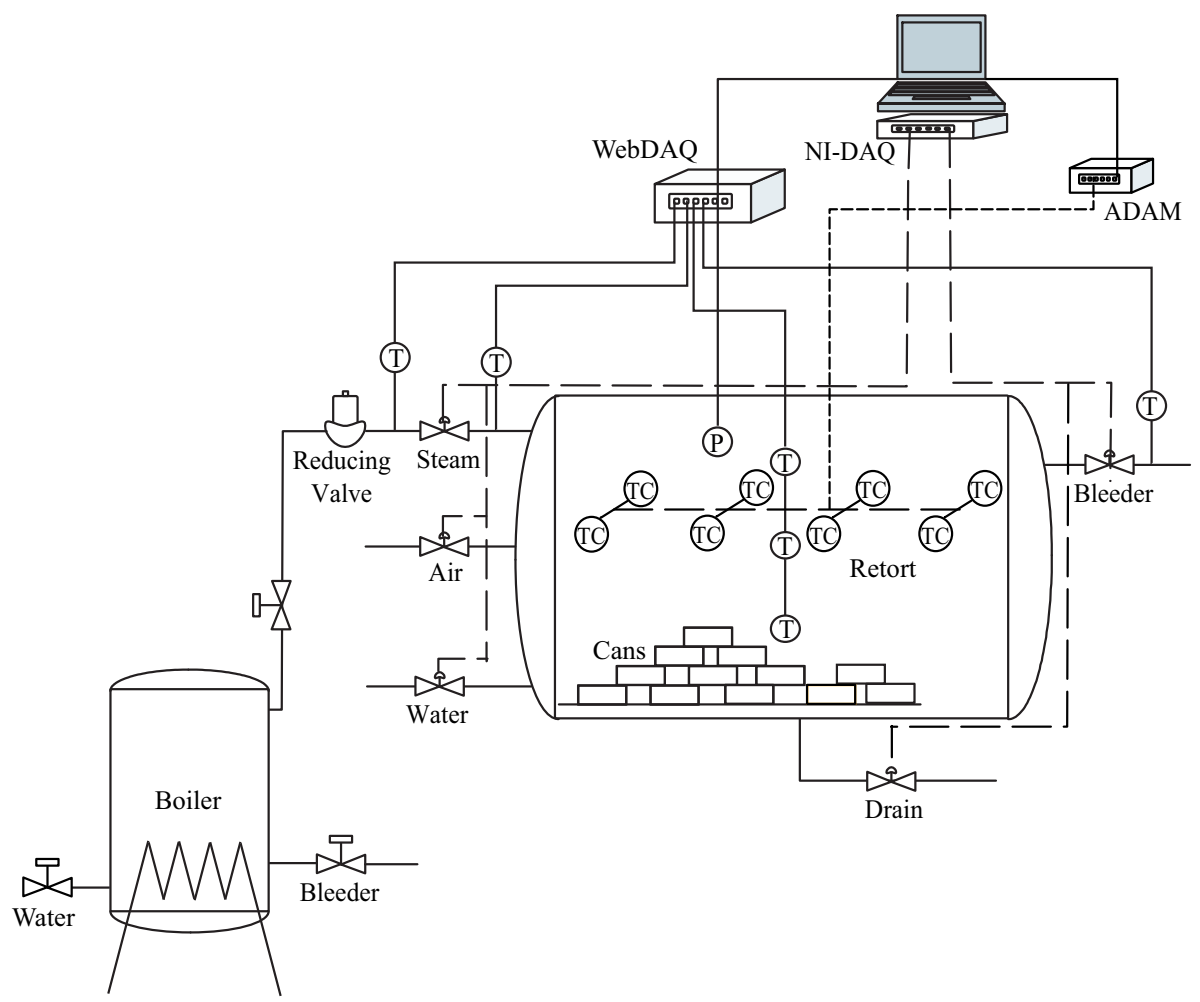

Figure 1: A prototype of thermal sterilization in a steam retort [8]

plant, the steam coming from the boiler is passed through a reduction valve 
in order to make the inlet steam valve pressure to be constant (in this case $P \sim 3.5$ bar $)$.

The retort temperature is measured by three resistance thermometers (PT100) located at the center cross section of the vessel and eight thermocouples (TC). The inlet steam, outlet bleeder and outlet drain temperatures are also measured with PT100 sensors while the pressure is measured with membrane transducers. The PT100 and pressure signals are sent to a external data acquisition module WebDAQ/100 which communicates with the PC through a $T C P / I P$ internet protocol while the thermocouple signals are sent to a external acquisition module $(A D A M)$ which communicates with the $\mathrm{PC}$ through a serial port. The monitorization and control is programmed in LabVIEW $W^{T M} 7$ Express. The signal is sent to the motorized valves (Siemens PV90(DN15)) through the internal data acquisition system (a digital input/digital output card $\left.N I-D A Q^{T M} 7\right)$.

From a control point of view, the system can be described as a Multi-input Multi-output MIMO plant, or more precisely, a set of MIMO plants representing the different stages of the process namely venting, heating and cooling. In the first two situations, inputs correspond with the positions of the steam, drain and purge valves, while the outputs are temperature and pressure inside the retort. The transition from venting to heating is detected by comparing current pressure measurements with the pressure corresponding to saturated steam which can be easily estimated from temperature measurements by applying the Antoine Law. In this way, when both variables (current pressure and saturated steam pressure) become the same, no air is present in the retort and heating may proceed. The system can be described by a set of ordinary differential equations, partial differential equations and algebraic equations, derived from mass and energy balances [8], [11]

\section{Neurofuzzy Models}

Takagi-Sugeno Fuzzy models (TS) [14] have been applied successfully in non-linear model based techniques [15]. These models may be formulated as an Adaptive Neuro-Fuzzy Inference System (ANFIS) [16]. In figure 2 an ANFIS is presented as an example with $\mathrm{N}$ input variables, one output variable and five layers. The first layer is composed of membership functions $A_{u i j}$, defined by the membership degree

$$
\mu_{A_{u i j}}: u_{i} \in \mathbb{R} \longmapsto \mu_{A_{u i j}}\left(u_{i}\right) \in \mathbb{R}
$$




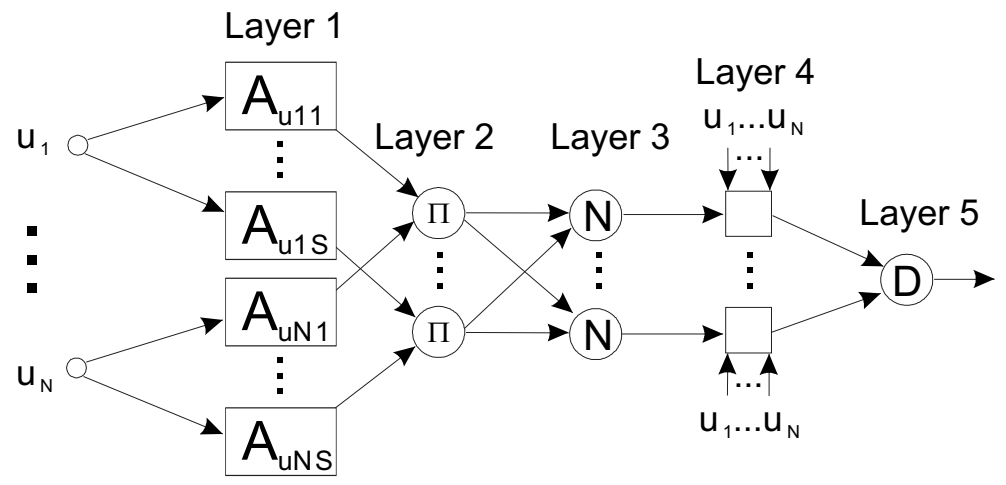

Figure 2: Fuzzy Neural Network [16]

The output of each node $i$ is $\mu_{A_{u i j}}\left(u_{i}\right)$, the membership degree of $u_{i}$. For the definition of these membership functions, some standard types are used. In this work, gaussian membership functions are used (see figure 4).

The second layer has nodes labelled with $\Pi$ which implement fuzzy inference machine. For example, if logical operation $A N D$ is carried out by multiplication, the output of each node $j$ of this layer is:

$$
\omega_{j}=\mu_{A_{u 1 j}}\left(u_{1}\right) \cdot \mu_{A_{u 2 j}}\left(u_{2}\right) \cdot \ldots \cdot \mu_{A_{u N j}}\left(u_{N}\right)
$$

The third layer normalizes the inference motor. The output of each node of this layer is:

$$
\overline{\omega_{i}}=\frac{\omega_{i}}{\sum_{i=1}^{N} \omega_{i}}
$$

The fourth layer has adaptive nodes:

$$
\overline{\omega_{i}} \cdot f_{i}=\overline{\omega_{i}} \cdot\left(p_{1 i} u_{1}+\ldots+p_{N i} u_{N}+r_{i}\right)
$$

Finally, the fifth layer is the defuzzyfication node. For TS systems, the output will be:

$$
\sum_{i=1}^{N} \overline{\omega_{i}} \cdot f_{i}=\frac{\sum_{i=1}^{N} \omega_{i} \cdot f_{i}}{\sum_{i=1}^{N} \omega_{i}}
$$

Fuzzy Neural Networks (FNN) combine the capability of uncertainty handling in information with learning skills [16], [17]. Recurrent Fuzzy Neural Networks (RFNN) have demonstrated to be better at getting all the dynamics of nonlinear systems. They are systems which have the same advantages 
as recurrent neural networks [18],[19]. RFNN are also named Fuzzy Dynamical Systems (see figure 3) and extend the application domain of FNN to temporal problems. Feedback allows the capture of dynamics and change. In the neurofuzzy model proposed by Takagi-Sugeno (TS), the structure of

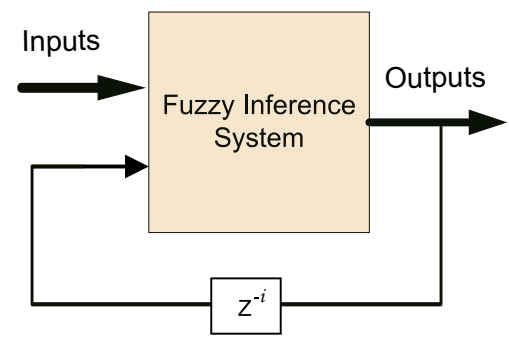

Figure 3: Dynamical Neurofuzzy System

antecedent describes fuzzy regions in the input space, and the one of consequent presents non-fuzzy functions of the model inputs. If recurrent functions with NARMAX structure (Non-linear Auto Regressive Moving Average with eXogenous input) of the kind:

$$
\hat{y}(k+1)=f(y(k), \ldots, y(k-m), u(k), \ldots, u(k-n))
$$

are used, being $u, y$ for each rule the inputs and outputs of the system respectively, the system may be described by the following way:

$R_{j}:$

IF $x_{1}(k)$ is $F_{1 j}, \ldots$, and $x_{n}(k)$ is $F_{n j}$,

THEN: $y_{j}(k)=a_{j}\left(z^{-1}\right) y(k-1)+b_{j}\left(z^{-1}\right) u(k-d)+c_{j}$

With $a_{j}\left(z^{-1}\right)=a_{1 j}+a_{2 j} z^{-1}+\ldots+a_{n_{y} j} z^{-\left(n_{y}-1\right)}$ and $b_{j}\left(z^{-1}\right)=b_{0 j}+b_{1 j} z^{-1}+$ $b_{2 j} z^{-2}+\ldots+b_{n_{u} j} z^{-n_{u}}$ and where $X(k)=\left[x_{1}(k) x_{2}(k) \ldots x_{n}(k)\right]^{T}$ is the input vector of the neurofuzzy system in the instant $k, F_{i j}$ is the fuzzy set respective to $x_{i}(k)$ on the rule $j, y_{j}(k)$ is the output of the model respective to the operating region associated to the rule, $d$ is the dead time and $c_{j}$ is a constant term. If $\mu_{i j}(k)$ is the membership degree of $x_{j}(k)$ in the fuzzy set $F_{i j}$ and the number of implications or rules is $L$, the RFNN complete model is possibly described by

$$
y(k)=\sum_{j=1}^{L} w_{j}(k)\left[a_{j}\left(z^{-1}\right) y(k-1)+b_{j}\left(z^{-1}\right) u(k-d)\right]+\xi(k)
$$


where

$$
w_{j}(k)=\frac{\bar{\mu}_{j}(k)}{\sum_{j=1}^{L} \bar{\mu}_{j}(k)}, \quad \bar{\mu}_{j}(k)=\prod_{i=1}^{n} \mu_{i j}(k), \quad \xi(k)=\sum_{j=1}^{L} w_{j}(k) c_{j}
$$

A study and comparison of fuzzy model based predictive control strategies, will be covered in this paper. Two strategies of NLMPC using neurofuzzy models will be presented, which, due to its low computational cost, will be suitable to implement on a typical industrial PLC of medium range, which is very common in industry. Moreover, taking into account that fuzzy technologies are being incorporated more and more in these kind of devices, which include specific libraries of functions, the programming and tuning of controllers will be simplified [2], [3].

In order to describe temperature profile in the heating period, a neurofuzzy model has been made. A previous knowledge of influence of variables in the temperature of autoclave and heuristic simulation expertise, have been used to obtain the inputs of the system, which were shown effective under computer simulations.

Inputs correspond with the positions of the steam valve $\left(u_{s}\right)$, drain and purge valves $\left(u_{d}, u_{b}\right)$, temperature of steam $\left(T_{s}\right)$ and previous samples for the temperature inside the retort $(T(t-1), T(t-2))$, while the output $T(t)$ is the current temperature of autoclave.

A real operation data set has been used in addition to simulation data obtained from a model derived from first principles and implemented in EcosimPro ${ }^{\circledR}[10],[11]$.

Subtractive clustering $(S C)$ method [12] has been used to provide initial rule parameter values. The training process to fit the FIS to dataset is based on a combination of the least-squares method and the backpropagation gradient descent method [13]. The rules base obtained is:

- IF $u_{s}(t)$ IS in1mf1 AND $T_{s}(t)$ IS in2mf1 AND $u_{b}(t)$ IS in3mf1 AND $u_{d}(t)$ IS in4mf1 AND $T(t-2)$ IS in5mf1 AND $T(t-1)$ IS in6mf1 THEN:

$$
\begin{array}{r}
T(t)=0.385 u_{s}(t)-0.141 T_{s}(t)+0.0584 u_{b}(t)+0.0875 u_{d}(t) \\
+0.125 T(t-2)+0.732 T(t-1)+0.116 ;
\end{array}
$$

- IF $u_{s}(t)$ IS in1mf2 AND $T_{s}(t)$ IS in2mf2 AND $u_{b}(t)$ IS in3mf2 AND $u_{d}(t)$ IS in4mf1 AND $T(t-2)$ IS in5mf2 AND $T(t-1)$ IS in6mf2 


\section{THEN:}

$$
\begin{aligned}
T(t)=0.0354 u_{s}(t)+ & 0.002 T_{s}(t)+0.007 u_{b}(t)+0.011 u_{d}(t) \\
& -0.308 T(t-2)+1.272 T(t-1)+0.015 ;
\end{aligned}
$$

where $\operatorname{in} i \mathbf{m f} j$ are the membership functions (MF) presented in figure 4. There is only one MF for input 4 (in4mf 1 ) because after the clustering method, two equal MF: in $4 \mathrm{mf} 1$ and in $4 \mathrm{mf} 2$ were produced. The meaning of MFs is not relevant to make the model. In fact, clustering methods frequently give arise to MFs difficult to interpret. In figure 5 the comparison between the model and the real data (open loop) of the temperature in the autoclave can be observed. The temperature shows a highly oscillatory behavior. This is due to the conversion to liquid of the steam inside the retort and other disturbance. As shown, the fuzzy model is able to accurately describe the system's behavior.

\section{Model based Predictive Control}

The control strategies presented here are based on one of the most popular algorithms: Generalized Predictive Control (GPC) [5]. The output prediction in GPC is given by a CARIMA (Controlled Auto-Regressive Integrated Moving Average) model of the plant:

$$
A\left(z^{-1}\right) y(t)=z^{-d} B\left(z^{-1}\right) u(t-1)+C\left(z^{-1}\right) \frac{\varepsilon(t)}{\Delta}
$$

where $u(t)$ and $y(t)$ are the control and output sequence of the system, $d$ the dead time of the plant, $\varepsilon(t)$ is a zero mean white noise

$$
\begin{gathered}
A\left(z^{-1}\right)=1+a_{1} z^{-1}+a_{2} z^{-2}+\ldots+a_{n a} z^{-n a} \\
B\left(z^{-1}\right)=b_{0}+b_{1} z^{-1}+b_{2} z^{-2}+\ldots+b_{n b} z^{-n b} \\
C\left(z^{-1}\right)=1+c_{1} z^{-1}+c_{2} z^{-2}+\ldots+c_{n c} z^{-n c}
\end{gathered}
$$

being $\Delta=1-z^{-1}$.

For simplicity, in the following $C\left(z^{-1}\right)$ is chosen to be 1 . The sequence of future control signals are calculated such that they minimize a multistage 

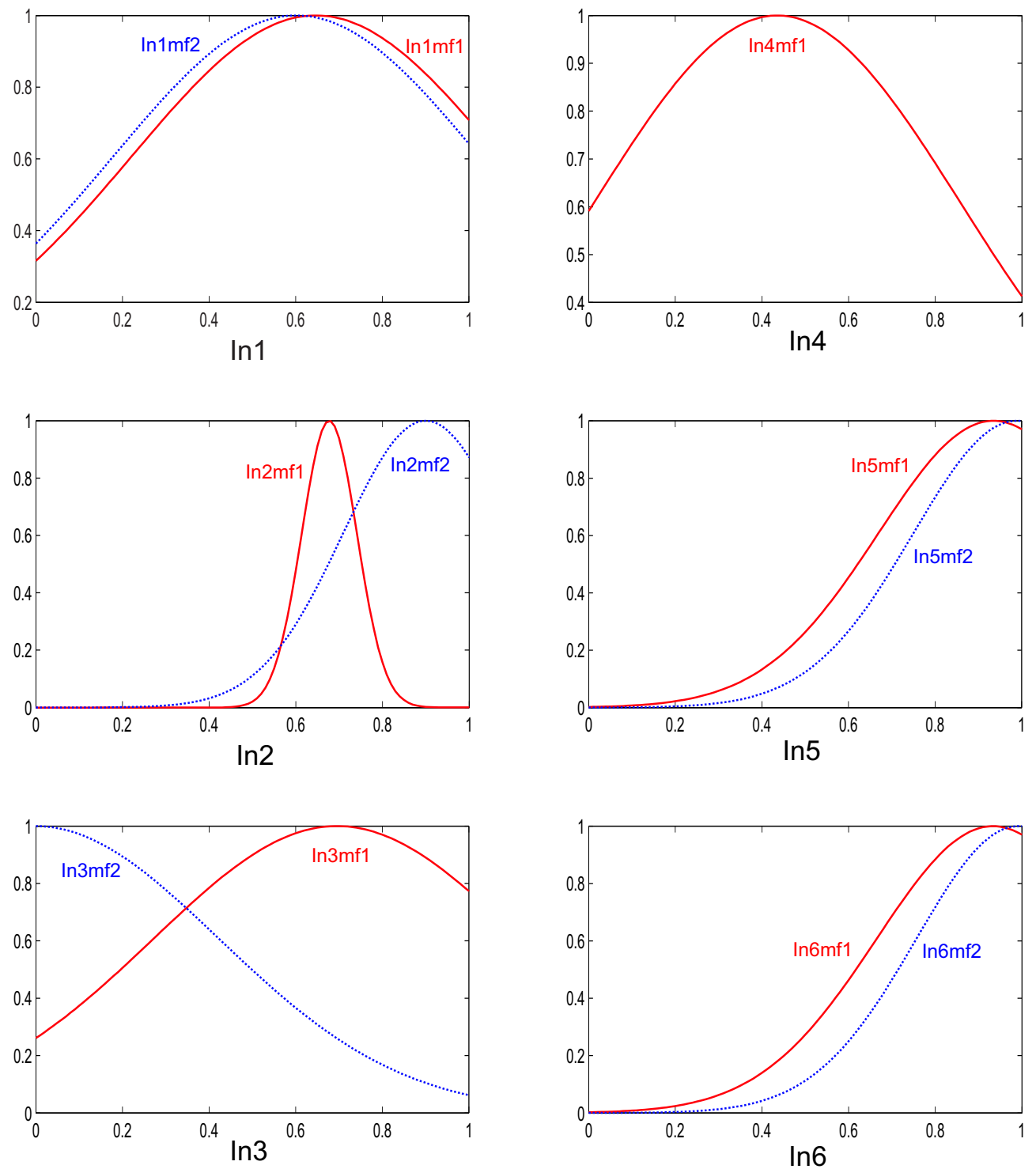

Figure 4: Membership functions of neurofuzzy system of autoclave 


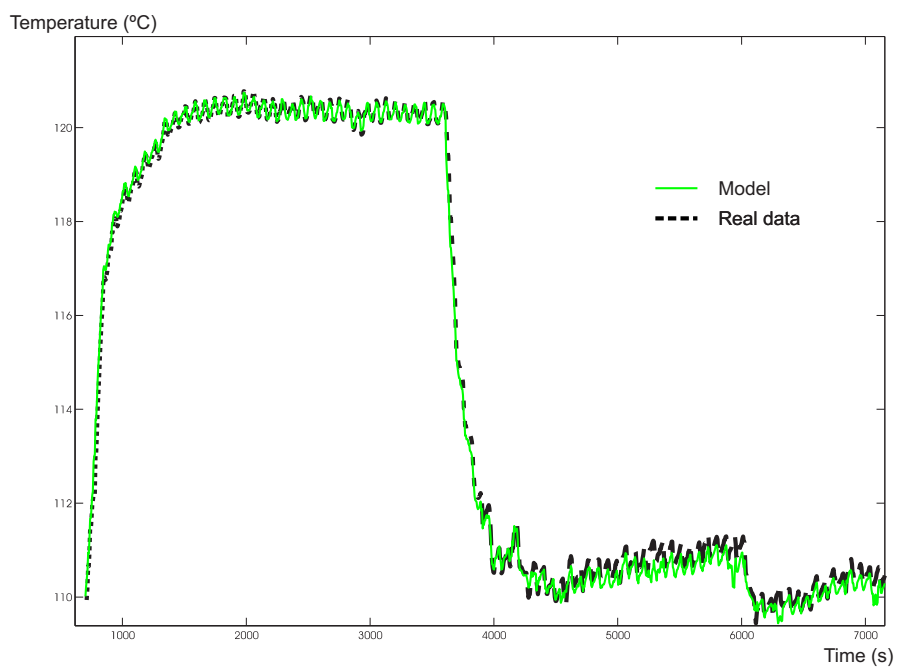

Figure 5: Validation of neurofuzzy model of the industrial autoclave

cost function defined by:

$J\left(N_{1}, N_{2}, N_{u}\right)=\sum_{J=N_{1}}^{N_{2}} \delta(j)[\widehat{y}(t+j \mid t)-\mathrm{w}(\mathrm{t}+\mathrm{j})]^{2}+\sum_{j=1}^{N_{u}} \lambda(j)[\Delta u(t+j-1)]^{2}$

Where $\widehat{y}(t+j \mid t)$ is a j-step ahead prediction of the system output on data up to time t, $N_{1}$ and $N_{2}$ are the minimum and maximum prediction horizon, $\delta(j)$ and $\lambda(j)$ are weighted sequences, and $\mathrm{w}(\mathrm{t}+\mathrm{j})$ is the future reference trajectory.

To solve the problem, we considered the following Diophantine equation:

$$
1=\Delta e_{j}\left(z^{-1}\right) A\left(z^{-1}\right)+z^{-j} f_{j}\left(z^{-1}\right)
$$

where $e_{j}\left(z^{-1}\right), f_{j}\left(z^{-1}\right)$ are polynomials uniquely defined. They can be obtained recursively in an easy way and they can be expressed by: $e_{j}\left(z^{-1}\right)=$ $e_{j, 0}+e_{j, 1} z^{-1}+e_{j, 2} z^{-2}+\ldots+e_{j, j-1} z^{-(j-1)}$, $f_{j}\left(z^{-1}\right)=f_{j, 0}+f_{j, 1} z^{-1}+f_{j, 2} z^{-2}+\ldots+f_{j, n_{y}} z^{-n_{y}}$,

In order to obtain the predictive output, let us first define the following expression:

$$
g_{j}\left(z^{-1}\right)=\Delta e_{j}\left(z^{-1}\right) B\left(z^{-1}\right)
$$

where $g_{j}\left(z^{-1}\right)=g_{j, 0}+g_{j, 1} z^{-1}+g_{j, 2} z^{-2}+\ldots+g_{j, j+N_{u}-1} z^{-\left(j+N_{u}-1\right)}$, 
and let us multiply equation (11) by $\Delta z^{j} e_{j}\left(z^{-1}\right)$ to get

$$
\Delta z^{j} e_{j}\left(z^{-1}\right) A\left(z^{-1}\right) y(k)=\Delta z^{j} e_{j}\left(z^{-1}\right) B\left(z^{-1}\right) u(k-d)+\bar{\xi}(k)
$$

where $\bar{\xi}(k)=\Delta z^{j} e_{j}\left(z^{-1}\right) \frac{\varepsilon(t)}{\Delta}$. Therefore by using (13) and (14), the following predictive output is obtained:

$$
\hat{y}(k+j \mid k)=f_{j}\left(z^{-1}\right) y(k)+g_{j}\left(z^{-1}\right) \Delta u(k+j-d)
$$

The horizon can be defined by $N_{1}=d+1, N_{2}=d+N$ and $N_{u}=N$. To solve the GPC problem, the set of control signals $\mathbf{u}=[u(t), u(t+1), \ldots, u(t+N)]^{T}$ has to be obtained in order to optimize the expression (12). As the cost function is quadratic, the optimum can be easily obtained, assuming there are no constraints on the control signals, making the gradient of $J$ equal to zero. Considering $\delta(j)$ and $\lambda(j)$ constants and grouping the terms of equation (16) which depend on the past, into $\mathbf{f}$, it leads to

$$
\mathbf{u}=\left(G^{T} G+\lambda I\right)^{-1} G^{T}(\mathbf{w}-\mathbf{f})
$$

where $G=\left[\begin{array}{llll}g_{d, 0} & g_{d+1,0} & \ldots & g_{N_{p}, 0}\end{array}\right]^{T}$

and $\mathbf{w}=\left[\begin{array}{llll}\mathrm{w}(\mathrm{t}+\mathrm{d}+1) & \mathrm{w}(\mathrm{t}+\mathrm{d}+2) & \ldots & \mathrm{w}(\mathrm{t}+\mathrm{d}+\mathrm{N})\end{array}\right]^{T}$ The control signal that is sent to the process is the first element of $\mathbf{u}$, given by:

$$
\Delta u(t)=\mathbf{K}(\mathbf{w}-\mathbf{f})
$$

\section{Fuzzy Generalized Predictive Control}

As mentioned in the introduction, linear controllers may result too poor on reference tracking when dealing with highly nonlinear systems. This fact will be illustrated in the following section by means of an experimental case study (the sterilization of solid food in steam retorts). In these cases, other alternatives, in which non-linear models in predictive control could be used, may result in suitable alternatives to the linear controllers. There are several proposals in this field [1], [20], [29] and [21]. An interesting option is to use neurofuzzy models [22],[23],[24],[25],[26],[27],[28]. Perhaps, the main issue in non-linear Predictive Control is how to obtain the optimizer solution, which consists in a non-convex problem and its resolution includes a high 
computational cost to solve in real time. During the last few years, several techniques have arisen to avoid the problems associated of an exact solution to the non-convex optimization problem [1]. Moreover, closed loop stability must be guaranteed.

Two strategies based on the neurofuzzy model obtained for autoclave are applied here. It is seen in section 3, the nonlinear model of the Takagi-Sugeno system, has a set of linear models described in equations 9 and 10. For simplification, supposing that $T_{s}, u_{b}$ and $u_{d}$ are constant for each rule, it can be written:

for rule 1:

$$
\Delta T(t)=T(t)-T(t-1)=0.385 \Delta u+0.125 \Delta T(t-2)+0.732 \Delta T(t-1)
$$

and for rule 2 :

$$
\Delta T(t)=T(t)-T(t-1)=0.0354 \Delta u-0.308 \Delta T(t-2)+1.273 \Delta T(t-1)
$$

the linear models equations for the controllers are:

for rule 1:

$T(t)=0.385 u(t-1)-0.385 u(t-2)+0.125 T(t-3)+0.606 T(t-2)+1.732 T(t-1)$

and for rule 2:

$T(t)=0.035 u(t-1)-0.035 u(t-2)-0.308 T(t-3)+1.580 T(t-2)+2.273 T(t-1)$

where $u(t)$ is the position of the steam valve.

A strategy proposed in [26], [28], [29] and [30] lies in calculating as many GPC controllers as linear models obtained in the neurofuzzy model, such that the controller output be:

$$
u(k)=\sum_{j=1}^{L} w_{j}(k) u_{j}(k)
$$

where $L$ is the number of linear models and $w_{j}$ was defined in $(8), u_{j}(k)$ is the control action given by equation (17), for each linear model of neurofuzzy model. This procedure will be referred to as the FGPC1. The advantage of this technique lies on its easy and fast implementation, which allows its programming on a simple industrial PLC. The main disadvantage of this strategy is that global optimum is not always found. However, a local optimum 
is guarantied for each rule or implication [30].

Another non-linear strategy based on neurofuzzy models (FGPC2) is proposed in [22]. Recurrent neurofuzzy model could be rewritten as a Linear Time Variant (LTV)

$$
\bar{a}\left(z^{-1}\right) y(k)=\bar{b}\left(z^{-1}\right) u(k-d)+\xi(k)
$$

Where $d$ is a transport delay, $\xi(k)$ is a white noise sequence with null average and

$$
\begin{aligned}
\bar{a}\left(z^{-1}\right) & =1-\bar{a}_{1} z^{-1}-\bar{a}_{2} z^{-2}-\ldots-\bar{a}_{n_{y}} z^{-n_{y}} \\
\bar{b}\left(z^{-1}\right) & =\bar{b}_{1} z^{-1}+\bar{b}_{2} z^{-2}+\ldots+\bar{b}_{n_{u}} z^{-n_{u}} \\
\bar{a}_{i} & =\sum_{j=1}^{L} w_{j}(k) a_{i j} z^{-i} \\
\bar{b}_{i} & =\sum_{j=1}^{L} w_{j}(k) b_{i j} z^{-i}
\end{aligned}
$$

The cost function defined in (12) can be expressed like:

$J(k)=(F y(k)+G \Delta u(k)+\Lambda-\Phi W)^{T}(F y(k)+G \Delta u(k)+\Lambda-\Phi W)+\left(\lambda\left(z^{-1}\right) \Delta u(k)\right)^{2}$

Where

$$
\begin{aligned}
F= & {\left[\begin{array}{llll}
f_{d}\left(z^{-1}\right) & f_{d+1}\left(z^{-1}\right) & \ldots & f_{N_{p}}\left(z^{-1}\right)
\end{array}\right]^{T}, } \\
\Lambda= & {\left[\begin{array}{lll}
\sum_{\rho=1}^{d+N_{u}-1} g_{d, \rho} \Delta u(k-\rho) & \sum_{\rho=1}^{d+N_{u}} g_{d+1, \rho} \Delta u(k-\rho) & \ldots \\
& \ldots \sum_{\rho=1}^{N_{p}+N_{u}-1} g_{N_{p}, \rho} \Delta u(k-\rho)
\end{array}\right]^{T} } \\
\Phi= & \operatorname{diag}\left\{\begin{array}{llll}
\delta_{d} & \delta_{d+1} & \ldots & \left.\delta_{N_{p}}\right\}
\end{array}\right. \\
W= & {\left[\begin{array}{llll}
w(k+d) & w(k+d+1) & \ldots\left(k+N_{p}\right)
\end{array}\right]^{T} }
\end{aligned}
$$

To reduce computational cost, control horizon is reduced to $N_{u}=1$. Which leads to

$$
G^{T}(F y(k)+\Lambda-\Phi W)+\left(G^{T} G+\lambda\left(z^{-1}\right) \lambda_{0}\right) \Delta u^{*}(k)=0
$$


To be able to simplify the control law, $\lambda_{0}^{2}=\lambda>0$ is chosen. Values $\lambda_{1}, \lambda_{2}, \ldots, \lambda_{N_{p}}$ are adjusted such that they meet:

$$
\Delta u^{*}(k)=\frac{G^{T}(\Phi W-F y(k))}{G^{T} G+\lambda} .
$$

\section{Control developments and experimental Results}

The pilot plant described in section 2 will be employed in this section in order to illustrate the performance of the different controllers, derived in this paper, on a real case study.

A previous control strategy using a PI-type controller parameterized by means of the Internal Model Control (IMC) technique [31] is presented on [8]. In the real plant, the controller has been readjusted, improving its performance.

For linear GPC, the model used was:

$$
\frac{T(z)}{u(z)}=\frac{0.01177 z-0.01182}{z^{2}-1.982 z+0.9821}
$$

Where $T(z)$ is the temperature inside the retort and $u(z)$ the opening of steam valve. After a tuning process over a simulation, the values chosen for the controller were: prediction and control horizons: $N_{p}=N_{u}=4$, weighting parameter of the control action $\lambda=0.6$. Using equation 18 :

$$
\begin{aligned}
u(k)= & u(k-1)-12.01 T(k-1)+21.68 T(k-2)-12.85 T(k-3)+2.30 T(k-4) \\
& +0.44 \mathrm{w}(k+1)+0.27 \mathrm{w}(k+2)+0.14 \mathrm{w}(k+3)+0.04 \mathrm{w}(k+4)
\end{aligned}
$$

Where a soft approximation of future reference trajectory $r(t+k)$ has been used with $\alpha=0.7$

$$
\mathrm{w}(t+k)=\alpha \mathrm{w}(t+k-1)+(1-\alpha) r(t+k), k=1, \ldots, N_{p}
$$

Figure 6 shows the closed loop response of the retort temperature (black line) with the PI and GPC controller. Four different steps have been introduced in the set point (green line). As illustrated in figure 6(a), the GPC controller is not only slow but it rarely reaches a good approximation to the set point. The PI controller has a better performance. In figures $6(\mathrm{~b}) /(\mathrm{c})$ we can observe a dependence of steam temperature $\left(T_{S}\right)$, which is a disturbance variable, on 

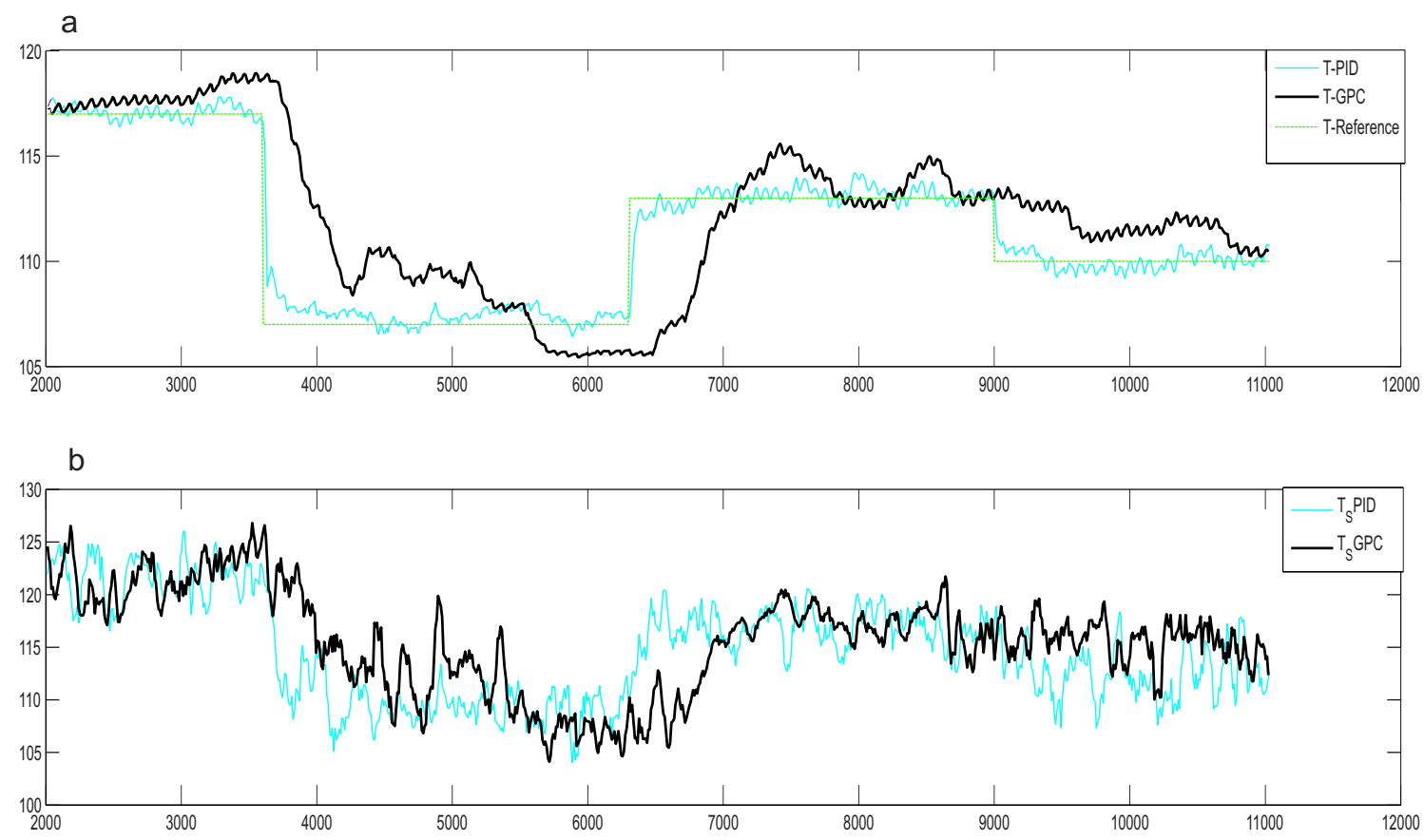

C

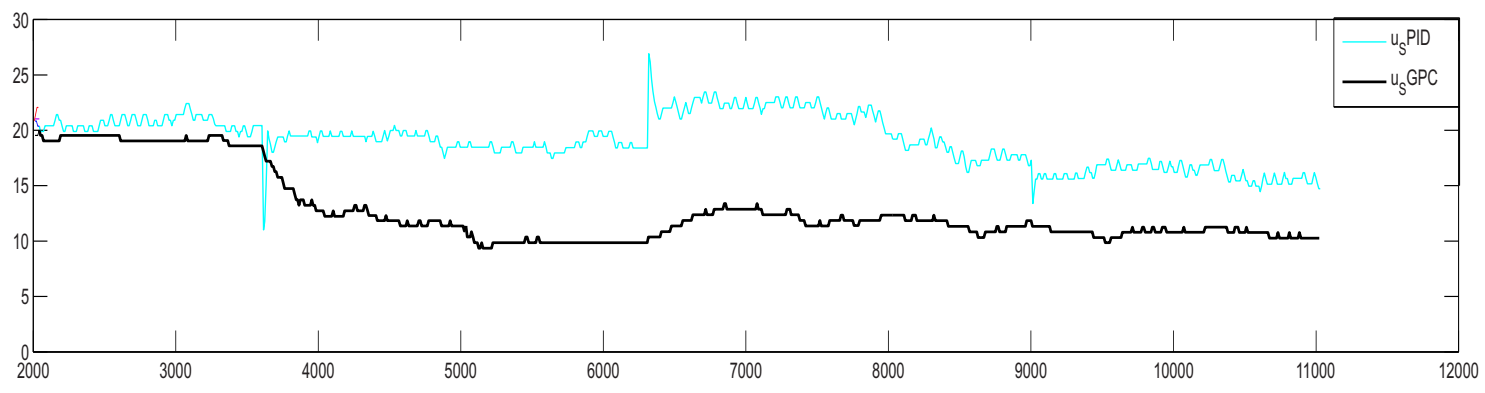

Figure 6: Performance of PI and GPC. (a) Tracking of temperature inside the retort. (b) Steam Temperature. (c) Steam valve opening percentage 
Table 1: Index comparison

\begin{tabular}{|c|c|c|c|c|}
\hline Index & PI & GPC & FGPC1 & FGPC2 \\
\hline$I A E$ & 3107 & 13901 & 5429 & 3975 \\
\hline$E_{t}$ & 107360 & 65741 & 58911 & 73907 \\
\hline
\end{tabular}

$u_{S}$. This is due to the change of the pressure produced by the valve in the steam pipeline. The PI controller is more aggressive in this change and the variation in $T_{S}$ helps to do better tracking than GPC. However, an MPC strategy will permit less energy consumption.

As mentioned in the introduction, a good controller performance is required in these kind of processes. In order to obtain a better closed loop response with less consumption, the nonlinear controller FGPC1 (see section 4) has been implemented. To carry out FGPC controllers proposed here using an industrial PLC, the gaussian membership functions (see fig. 4) are changed by new triangular membership function. In figure 7 , the new equivalent functions are shown. This is due to many PLC only have triangular functions

The comparison between FGPC and GPC controllers is presented in Figure 8. It is clear that this control scheme is able to approach the retort temperature (black line) to the set point (green line) better than the GPC (blue line).

Comparison between FGPC and PI behavior is shown in figure 9. We can observe a better tracking of PI, but with more energy consumption In a third test, the FGPC2 technique has also been applied to the pilot plant under similar conditions than the other controller. Black line in Figure 10 corresponds with the closed loop (FGPC2) temperature response. At first sight it seems (although difficult to assert) that it improves the FGPC1 results. In order to obtain a more quantitative comparison between these strategies, the IAE (as a measurement of the tracking error) and an index of energy consumption $E_{t}=\int_{t} u_{s}(t) d t$. have been computed and presented in Table 1. The IAE for the FGPC2 is about $27 \%$ lower than the IAE for the FGPC1 asserting that its performance is better.

It is important to highlight that the FGPC2 presents a similar performance to the PI, but with a savings of approximately $41 \%$ of energy. In figure 11 , 

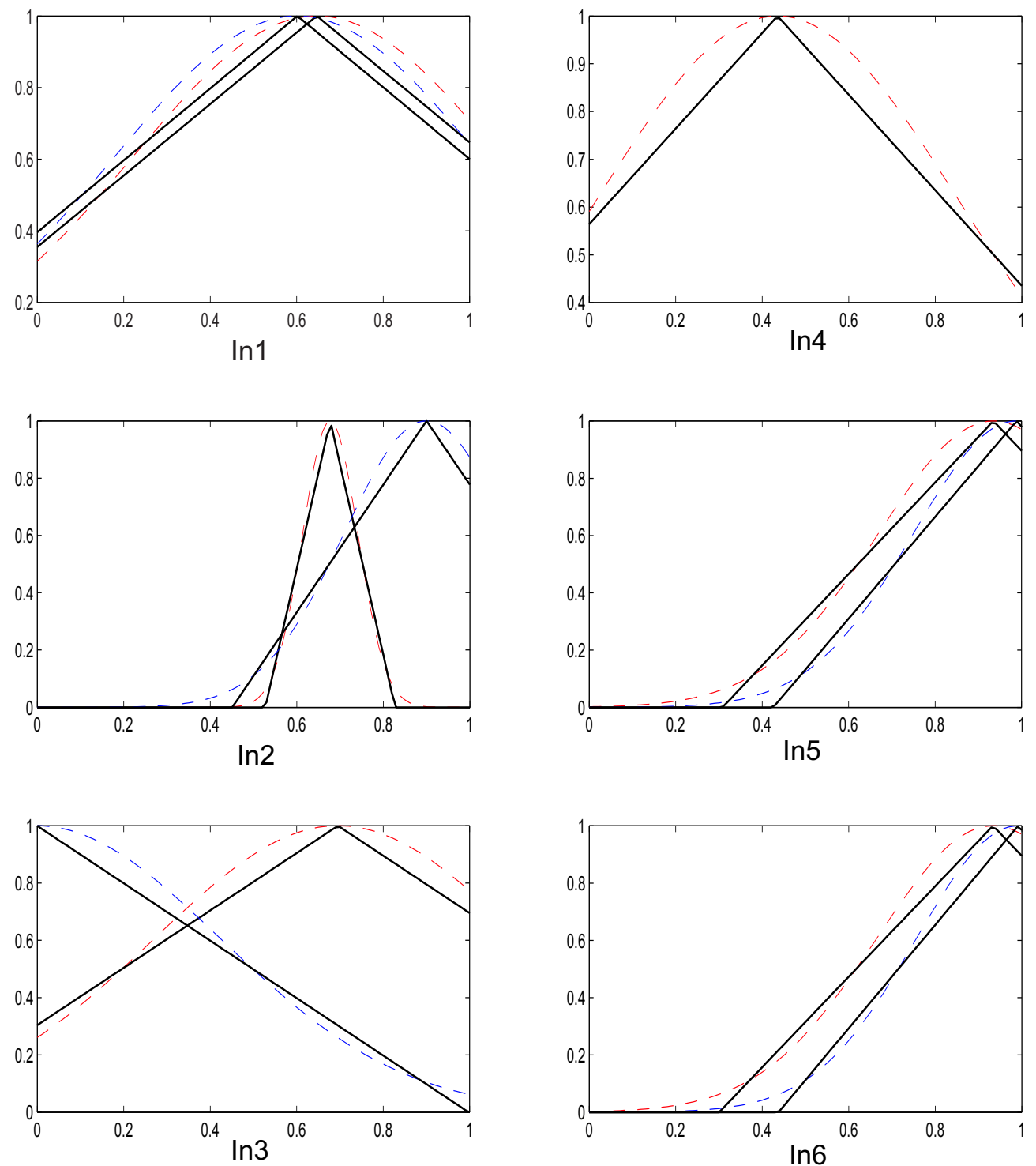

Figure 7: Equivalent triangular membership functions 


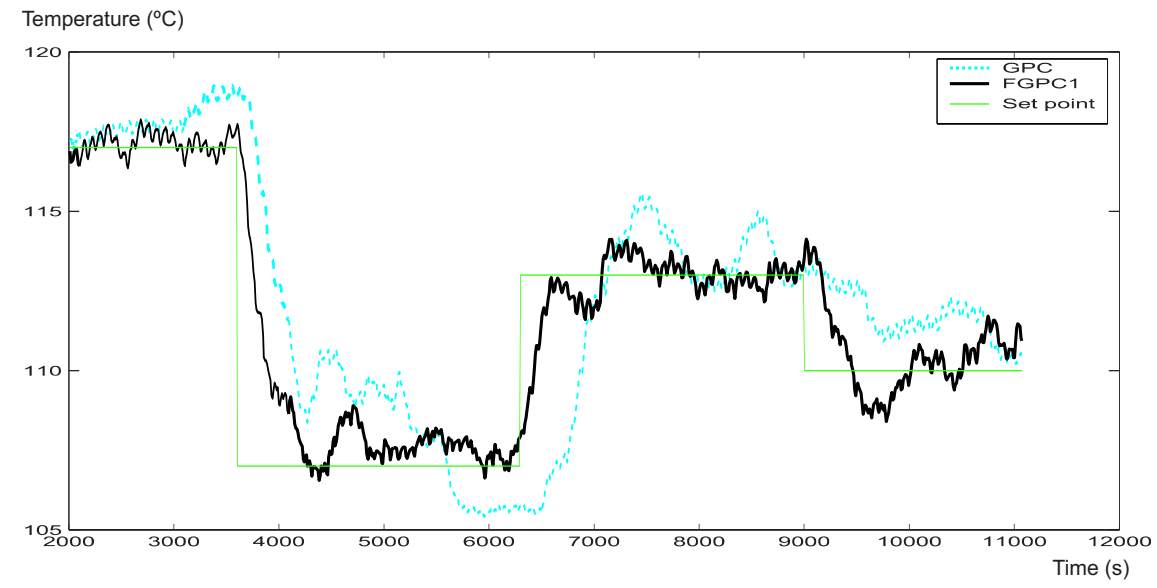

Figure 8: Comparison between Predictive Control strategies Non-linear vs linear

we can compare all the strategies presented.

\section{Conclusions}

Three MPC control strategies (one linear and two nonlinear) have been designed and implemented in a pilot plant for the thermal sterilization of solid canned food in steam retorts. The nonlinear controllers have been based on Neurofuzzy models which have been validated with experimental data from the real plant. A very good agreement between experimental and simulated data by the model has been obtained even for a large prediction horizon. The controller with the best performance has been the Non-linear GPC based on a recurrent Fuzzy Network LTV. Both nonlinear strategies present much better behavior than linear MPC strategy based on GPC. In comparison with a PI controller parameterized by means of the IMC technique [8], the second NLMPC strategy presents less energy consumption with similar tracking. The main advantage of these non-linear strategies is the possibility of being carried out on a PLC, saving a considerable cost in hardware; no distributed control systems (DCS) or expensive comercial hardware platforms are needed. This, in addition to modelling simplicity, makes it very attractive to the implementation into an industrial environment. 


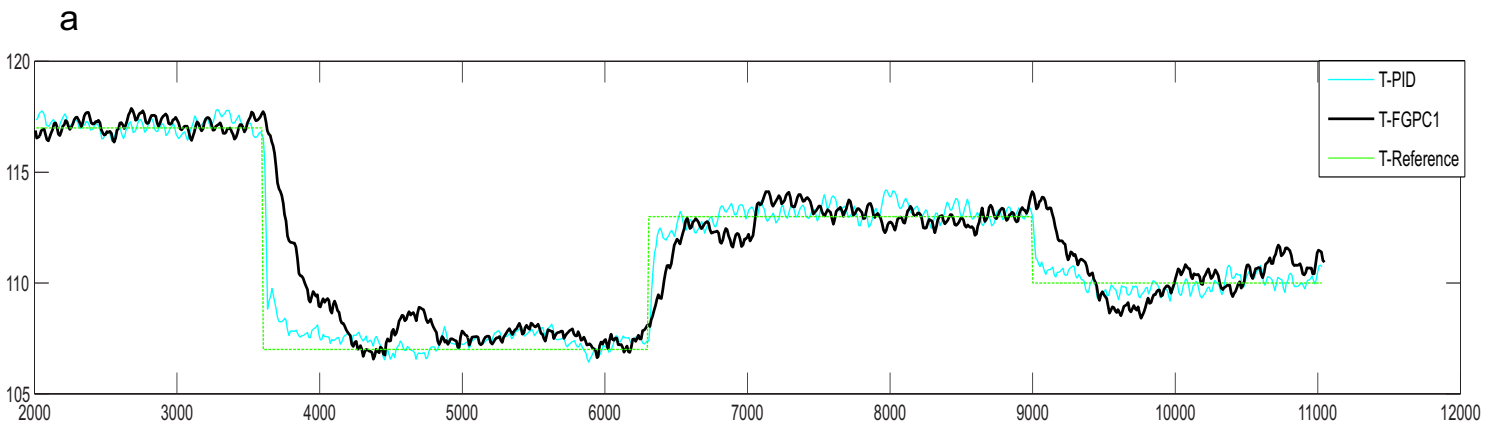

b
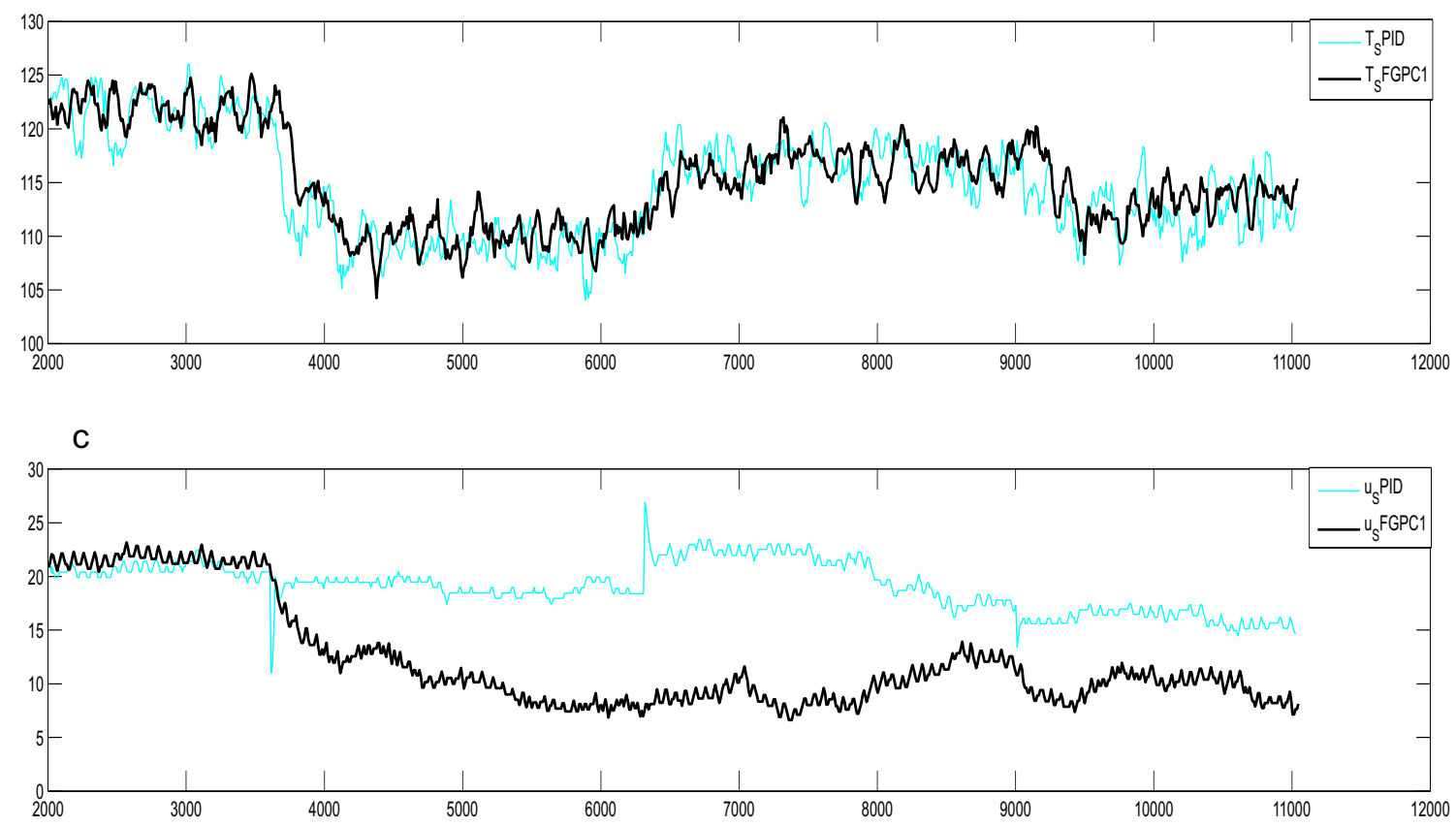

Figure 9: Comparison between PI and FGPC1. (a) Tracking of temperature inside the retort. (b) Steam Temperature. (c) Steam valve opening percentage 

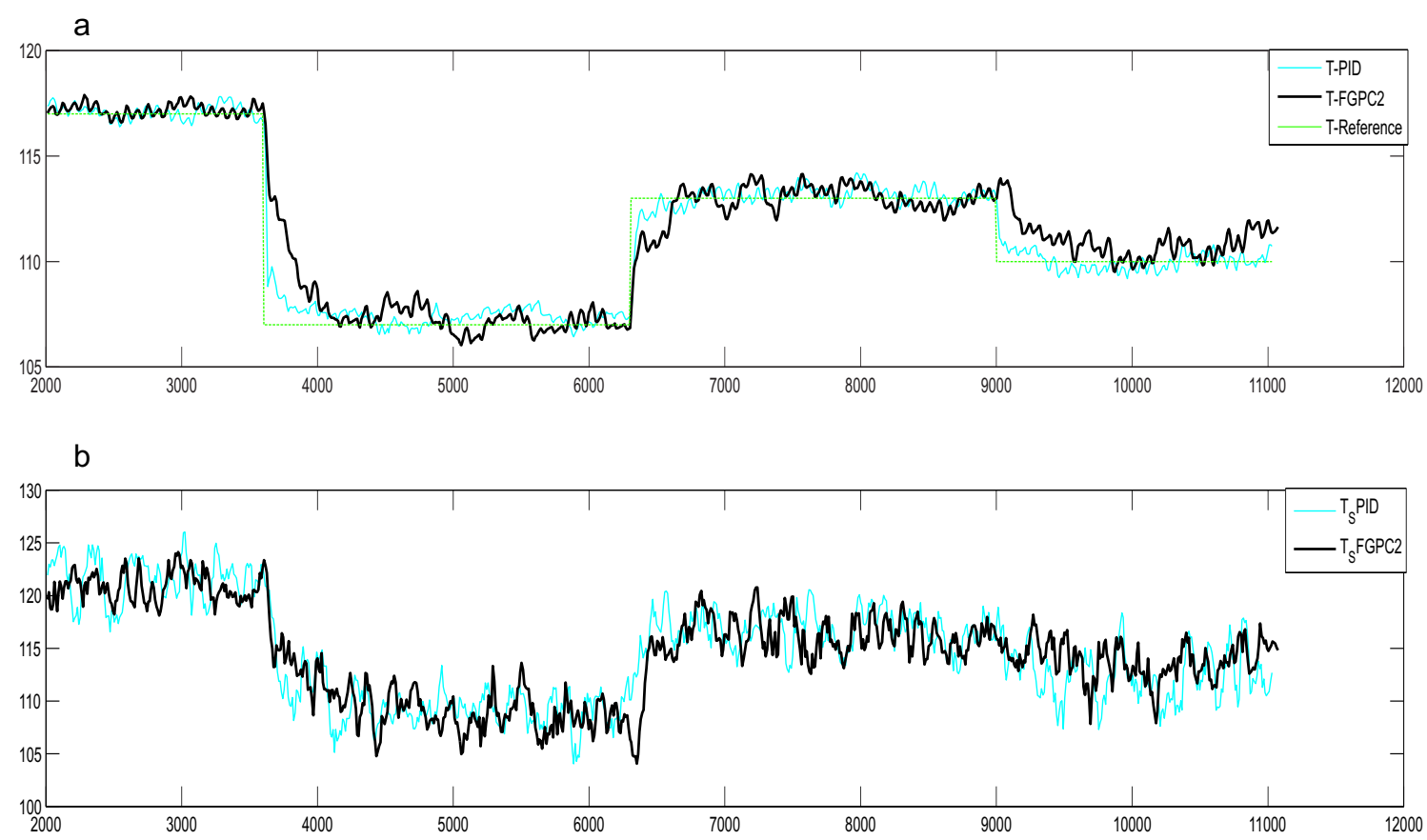

C

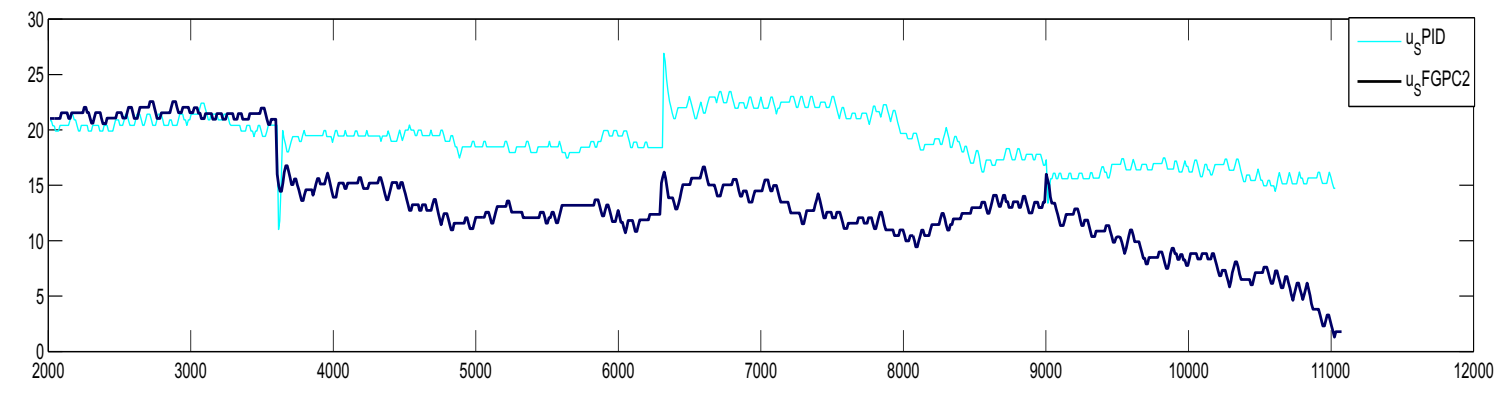

Figure 10: Comparison between PI and FGPC2. (a) Tracking of temperature inside the retort. (b) Steam Temperature. (c) Steam valve opening percentage 

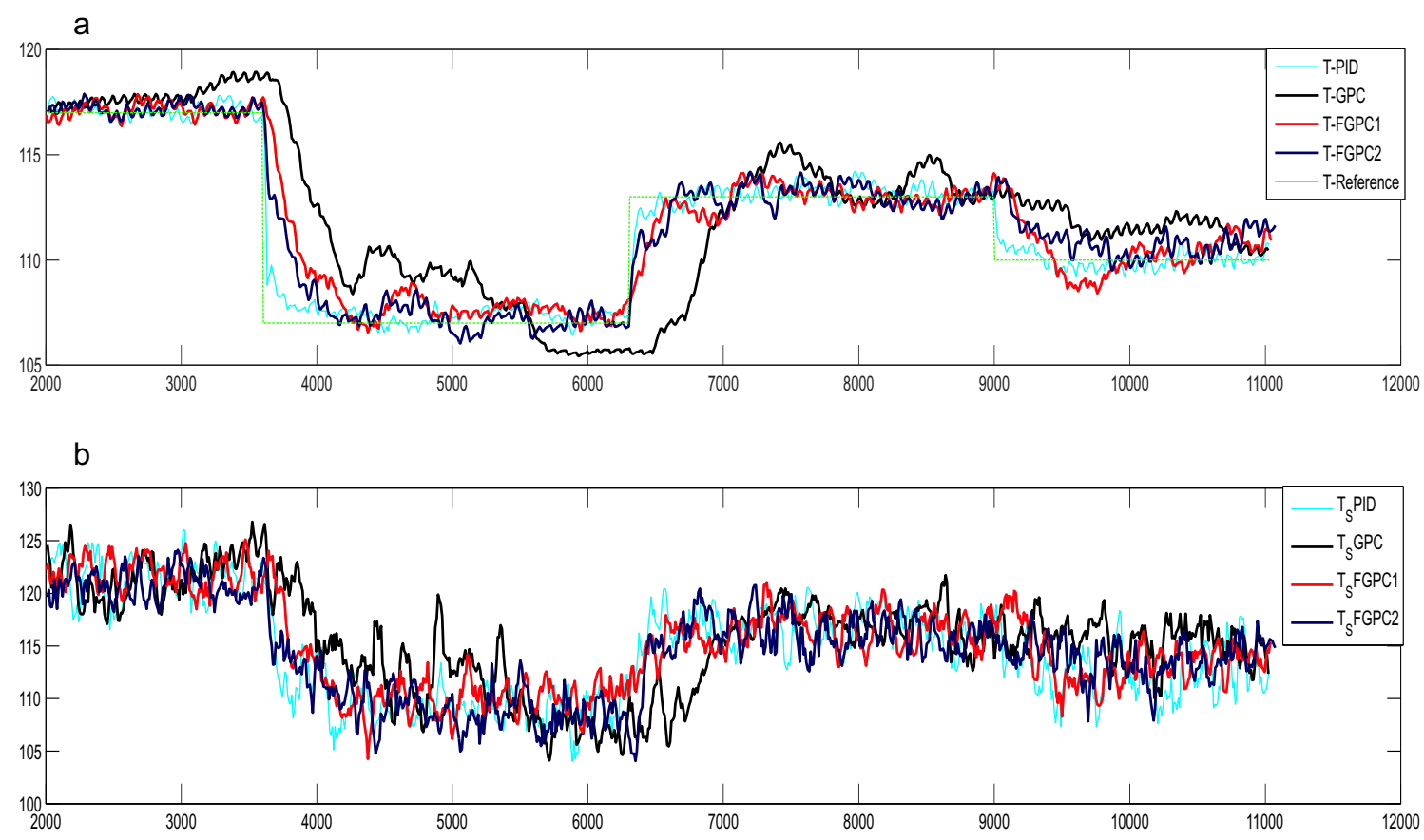

C

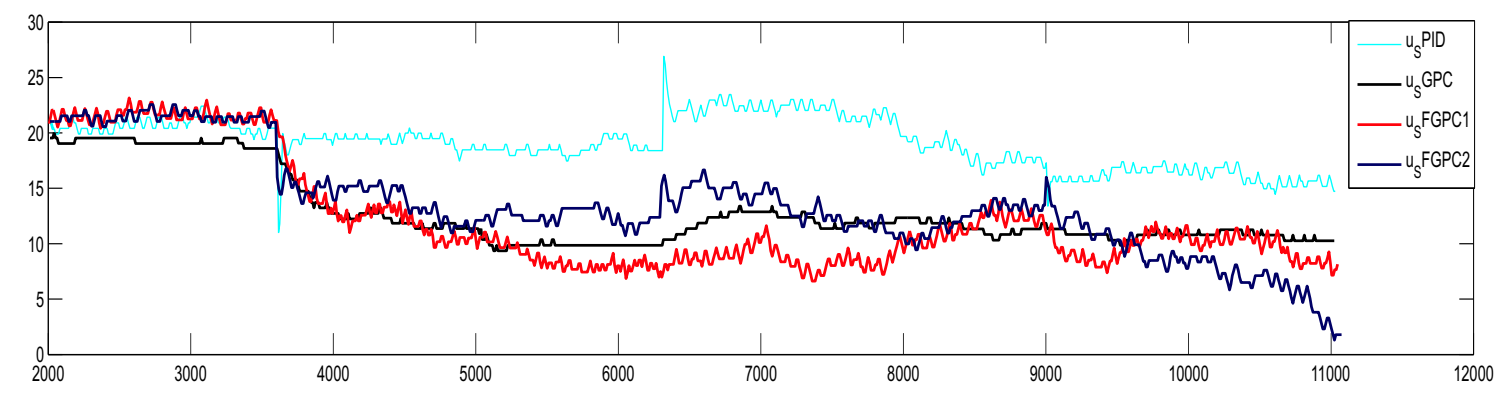

Figure 11: Comparison between PI, GPC, FGPC1 and FGPC2. (a) Tracking of temperature inside the retort. (b) Steam Temperature. (c) Steam valve opening percentage 


\section{Acknowledgement}

The authors acknowledge MCYT-Spain for funding this work under grants DPI2004-07444-C04 and DPI2007-66718-C04-01

\section{References}

[1] E.F. Camacho, C. Bordons. "Model Predictive Control", SpringerVerlag, London (2004)

[2] INFORM (Institut für Operations Research und Management) GmbH. http://www.fuzzytech.com

[3] International Electrotechnical Commission (IEC) "IEC 1131 Programable Controllers. Part 7: Fuzzy Control Programming" IEC TC65/WG 7/TF8 (1997)

[4] International Electrotechnical Commission (IEC). " "Programmable Controllers Part 3: Programming Languages, IEC 61131-3, second edition" IEC TC65/SC65B/WG7/TF3 (1998)

[5] D.W. Clarke, C. Mohtadi, P.S. Tuffs. "Generalized redictive Control. Part I. The basic algrithm" Automatica, 23(2), pp 137-148 (1987)

[6] A.A. Alonso, R.I.P. Martín, N.V. Shukla, P.B. Deshpande. "On-line Quality Control of (Non-linear) Batch Systems: Application to Thermal Processing of Canned Foods", Journal of Food Engineering 19, pp 275289 (1993)

[7] A.A. Alonso, J.R. Banga, R.I.P. Martín. "A New Strategy for the Control of Pressure During the Cooling Stage of the Sterilization Process in Steam Retorts", Food and Bioproducs Processing 71, pp 197-205 (1993)

[8] A.A. Alonso, J.R. Banga, R. Pérez-Martín. "A complete dynamic model for the thermal processing of bioproducts in batch units and its application to controller design", Chemical engineering science 52, pp 130\%1322 (1997)

[9] A.A. Alonso, J.R. Banga, R.I.P. Martín. "Modeling and Adaptive Control of a Batch Sterilization Process" Computers and Chemical Engineering. 22(3), 445-458 (1998) 
[10] EcosimPro by EA Internacional. http://www.ecosimpro.com

[11] C. Vilas, M. R. García, J. R. Banga, A. A. Alonso "Desarrollo de una librería de componentes en ecosimpro para la operación de plantas de procesamiento térmico de alimentos" (in Spanish) Revista Iberoamericana de Automática e Informática Industrial (RIAI)5(1), pp.51-65 (2008)

[12] S. Chiu, "Fuzzy model identification based on cluster estimation", Journal of Intelligent and Fuzzy Systems, vol. 2, no.3, pp. 267-278 (1994)

[13] J.-S.R. Jang, "ANFIS: Adaptive-network-based fuzzy inference system" Systems, Man and Cybernetics, IEEE Transactions on Volume 23, Issue 3, Page(s):665 - 685 (1993)

[14] T. Takagi and M. Sugeno, "Fuzzy identification of systems and its applications to modelling and control" IEEE Trans. Syst., Man, Cybern. 15, pp. 116-132 (1985)

[15] K. Kiriakidis, "Fuzzy Model-Based Control of Complex Plants" IEEE Transactions On Fuzzy Systems 6(4), pp. 517-529 (1998)

[16] J.S.R. Jang, C.T. Sun, E. Mizutani. "Neuro-fuzzy and Soft Computing" Prentice-Hall (1997)

[17] R. Fullér. "Neural Fuzzy Systems" Åbo Akademis tryckeri, Åbo (1995)

[18] C.-C. Ku, K.Y. Lee "Diagonal recurrent neural networks for dynamic systems control" IEEE Transactions on Neural Networks 6(1), pp 144156 (1995)

[19] C.-H. Lee, C.-C. Teng "Identification and Control of Dynamic Systems Using Recurrent Fuzzy Neural Networks" IEEE Transactions On Fuzzy Systems 8(4), pp 349-366 (2000)

[20] B. Kouvartitakis, M. Cannon, J.A. Rossiter. "Non-linear model based predictive control" International Journal of Control 72(10), pp 919-928 (1999)

[21] T.A. Johansen, J.T. Evans, B.A. Foss. "Identification of nonlinear system structure and parameters using regime decomposition" Automatica 31(2), pp 321-326 (1995) 
[22] Chi-Huang L., Ching-Chih T. "Generalized predictive control using recurrent fuzzy neural networks for industrial processes" Journal of Process Control 17, pp 83-92 (2007)

[23] S. Blazic, I. Skrajanc. "Design and stability analysis of Fuzzy Modelbased Predictive Control -A case study" J Intell Robot Syst. Springer Science (2007)

[24] M. Mahfouf, D.A.Linkens, M.F. Abbod "Adaptive Fuzzy TSK Modelbased Predictive Control using a CARIMA model structure" Institution of Chemical Engineers, Trans IChemE, Vol 78, Part A (2000)

[25] H.N. Nounou, K.M. Passino "Fuzzy model Predictive Control: Techniques, Stability Issues, and Examples" Proc. IEEE International Symposium on Intelligent Control/Intelligent Systems and Semiotics, pp. 423-428 (1999)

[26] J. Zhang, A.J. Morris "Long Range Predictive Control of nonlinear processes based on recurrent Neuro-Fuzzy network models" Neural Computing and Applications. Springer-Verlag London (2000)

[27] J.J. Espinosa, J. Vandewalle. "Predictive Control using Fuzzy models" Advances in Soft Computing in Engineering Design and Manufacturing (1998)

[28] J.J. Espinosa, M.Hadjili, V. wertz, J. Vandewalle. "Predictive Control using Fuzzy models-Comparative study" Proc. European Control Conference (1999)

[29] S. Townsend, G.W. Irwin "Nonlinear model based predictive control using multiple local models" Nonlinear Predictive Control. IEE Control Engineering series (2001)

[30] J. Soto, I. Castillo, A. Gauthier, V. Grisales. "Identificación Y control De sistemas no lineales mediante Clustering y GPC usando modelos difusos Takagi-Sugeno"(in Spanish) Revista de Ingeniería 19, pp. 149-156. F. de Ingeniería, Universidad de los Andes (2006)

[31] M. Morari, E. Zafiriou "Robust process control" Prentice-Hall (1989) 